\title{
Proposal of a Design Methodology for Modularity Using Geometric Information
}

\author{
Keita TONOIKE ${ }^{1}$, Shinichi FUKUSHIGE ${ }^{1}$, Yasushi UMEDA ${ }^{1}$ \\ ${ }^{1}$ Department of Mechanical Engineering, Graduate School of Engineering, Osaka University, Japan, \\ tonoike@lce.mech.eng.osaka-u.ac.jp
}

\begin{abstract}
:
In these days, modular design of a product is becoming important even for environmental conscious design in order to improve, e. g., reusability, disassemblability, and recyclability. In order to derive a feasible module structure, not only environmental aspects but also geometric information bears important roles. This paper presents a modular design methodology for life cycle engineering that proposes module structure by using information on geometry and product life cycle. The case study of an inkjet printer revealed that the derived modular structure satisfies the various demands from product life cycle with keeping the geometric consistency.
\end{abstract}

Keywords: Design for Environment, Modular Design, SOM, Geometry, Life Cycle Option

\section{Introduction}

Modular design has been studied for improving, e. g., manufacturability and assemblability. In these days, modular design is also becoming important for environmental conscious design in order to improve, e. g., reusability, disassemblability, and recyclability. In order to derive a feasible module structure of a product, we should consider both of life cycle options, such as reuse and recycling, and structure of the product, such as component configuration, position and shape of components. This paper proposes a method to determine appropriate modular structure based on these factors. First, the method calculates similarity of attributes of components such as materials, physical life, value life, and life cycle options by using Self-Organizing Map (SOM) and classifies the components. As a result, the components are classified into some groups that should trace the same life cycle and, therefore, each group should form a module. Next, the method derives physically plausible module structure by dividing the groups of the components based on geometric information and connectivity of components.

\section{Related Works}

In general, modularity is an approach that supports the handling of complex systems by structuring them into several independent sub-systems [1, 2,].

Some existing methodologies focus on the evaluation of relations between functional carriers, e.g. energy and material flow, such as Ishii [3], the heuristic approach from Stone, et al. [4] and the Design Structure Matrix from Pimmler and Eppinger [5].

Kusiak and Huang offer the possibility to group functional carriers to modules from the viewpoint of production [6]. This methodology points in the right direction but focuses only on production.

For supporting environmentally conscious design, modular design should consider both of the environmental aspect and the product structure including geometry. However, as discussed above, there are no existing methodologies that deal with both kinds of information.

\section{Approach for Modular Design \\ 3.1 Classification}

First, we calculate similarity of components' attributes related to environmental consciousness of a product, by using Self-Organizing Map (SOM) [7] and classify the components. In this research, we employ material kinds, physical life, value life, and life cycle options as the attributes of components.

The Self-Organizing Map (see Fig. 1) is a computational method for visualizing and analyzing similarity of high-dimensional data. SOM autonomously finds out a projection from a set of given data items onto a regular grid. This flexibility and autonomy are the reason why we use SOM for classification in this study. As a result, the components are classified into some groups that should trace the same life cycle and, therefore, each group should form a module.

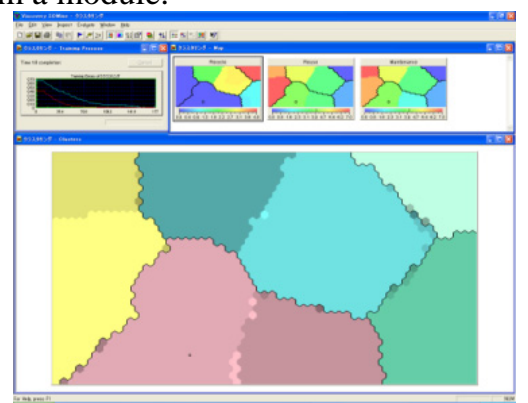

Fig. 1: Self-Organizing Map (SOM)

\subsection{Dividing the groups into modules}

Next, we derive physically plausible module structure by dividing each group of the components into some modules, based on geometric information and connectivity of components. In this study, we use three-dimensional geometry of components and product structure as the geometric information. For representing geometric suitability of a module, we define "module density" as a rate of volumes of components to a convex hull of those components that will be included in a module. We assume 
that the module with higher module density is better.

The convex hull for a set of objects in a three-dimensional space is the minimal convex set containing all objects. Here, we define approximate module structure of several components as follows. For example, given four components to be modularized, six convex hulls of component pairs are extracted as shown in Fig. 2 (a). Among these convex hulls, simply connected space of convex hulls, which contains all components, represents the approximate module structure as shown in Fig. 2 (b). Section 4 will describe how convex hulls are selected to construct the approximate module structure.

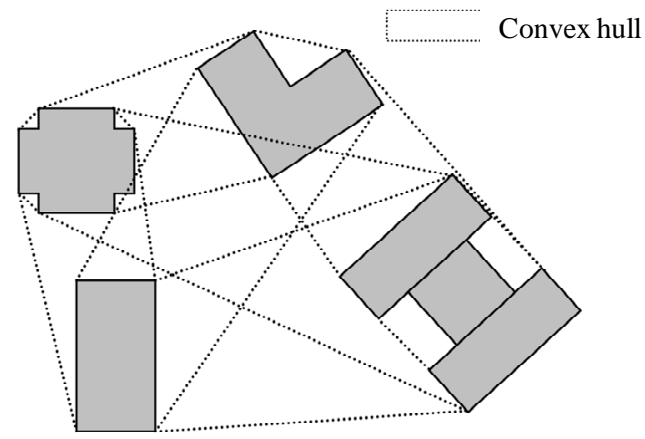

(a)Convex hulls

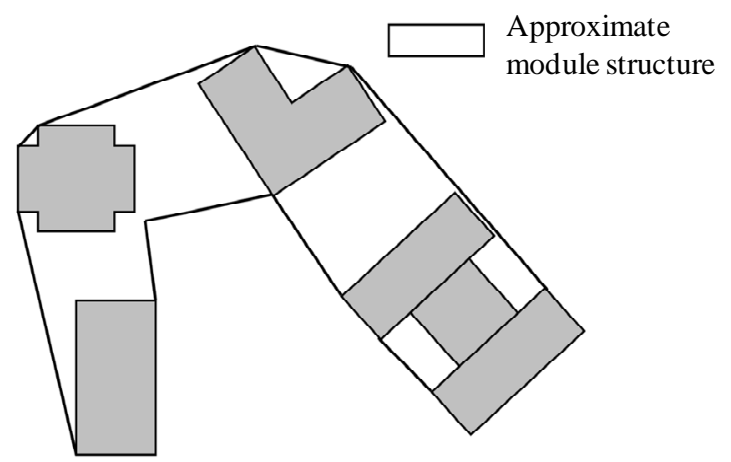

(b)Approximate module structure

Fig. 2: Convex hulls and approximate module structure

As proposed above, we assume that the module with higher module density is better (see Fig. 3). The module density is regarded as a normalized distance between components. This means that if the module density is higher, the components included in the module are closer to each other. Therefore, such module is stable from the geometric viewpoint.

The volume of components is given and never changes in this study. Then, maximizing the module density means minimizing the sum of the volumes of all modules. This minimization leads to suppressing expansion of size of the whole product, which is a demerit of the modular design.

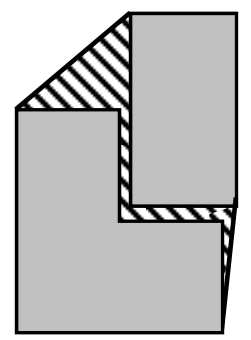

(a)High module density

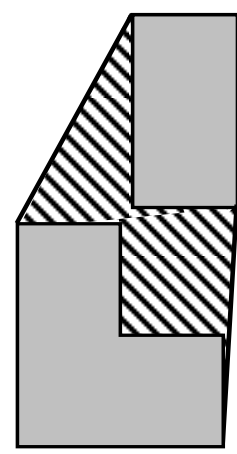

(b)Low module density
Fig. 3: Convex hull and module density

\subsection{Evaluation of modularity}

We evaluate the structure of modules quantitatively by using the modular density and connectivity of components.

We define the evaluation value Combination $\left(x_{i}, x_{j}\right)$ of a combination of two components $x_{i}$ and $x_{j}$, which belong to a same group described in Section 3.1, as follows:

$$
\begin{aligned}
\operatorname{Combination}\left(x_{i}, x_{j}\right)= & \operatorname{Density}\left(x_{i}, x_{j}\right) \times(1-\alpha) \\
& +\operatorname{Connection}\left(x_{i}, x_{j}\right) \times \alpha
\end{aligned}
$$

Where $\alpha$ is a weight between the module density and the connectivity. The module density $\operatorname{Density}\left(x_{i}, x_{j}\right)$ is defined as:

$$
\operatorname{Density}\left(x_{i}, x_{j}\right)=\frac{V\left(x_{i}\right)+V\left(x_{j}\right)}{V\left(H\left(x_{i}, x_{j}\right)\right)}
$$

Where, $H\left(x_{i}, x_{j}\right)$ is a convex hull of components $x_{i}$, and $x_{j}$. $V\left(x_{i}\right), V\left(x_{j}\right)$ and $V\left(H\left(x_{i}, x_{j}\right)\right)$ are the volume of $x_{i,}, x_{j}$ and $H\left(x_{i}, x_{j}\right)$, respectively.

Connection $\left(x_{i}, x_{j}\right)$ represents the connectivity between components $x_{i}$, and $x_{j}$ as follows:

$$
\text { Connection }\left(x_{i}, x_{j}\right)= \begin{cases}1 & \left(x_{i} \text { and } x_{j}\right. \text { are } \\ 0 & \text { directly connected })\end{cases}
$$

(else)

Then, we define the following value Modularity for a module structure. In other words, the best module structure is the one that has its highest value.

$$
\text { Modularity }=\sum_{k=1}^{N} \sum_{P_{i j} \in M_{k}} \text { Combination }\left(x_{i}, x_{j}\right)
$$

Where, $N$ is the number of modules in the product. $M_{k}$ is $k$ th module in the product and $P_{i j}$ represents a pair of components $x_{i}$ and $x_{j}$ in the module $\mathrm{M}_{\mathrm{k}}$. Pairs $P_{i j}$ of components are the pairs that compose the approximate module structure described in Section 3.2.

\subsection{Constraints for module structure}

In this method, we employ two constraints for constructing the module structure. First, as discussed above, a module should consist of components in a same group. Violating this constraint may drastically decrease reusability and recyclability. And second, a module should not geometrically surround a component of a different group. Since in such a situation the component cannot be 
extracted without disassembling the module, violation of this constraint also decreases disassemblability, reusability, and recyclability. The geometric information is employed to avoid such a situation. The way to judge inclusion of a component as follows. A component is approximated as a cube and the cube is extended to six directions. If all of them are interacted other components which consists of a module, the component is included in the module. Fig. 4 shows an example of such a situation. There are four components A, B, C, and D. The component D is in a different group. The components $\mathrm{A}, \mathrm{B}$, and $\mathrm{C}$ should not be modularized, because $\mathrm{D}$ cannot be extracted.

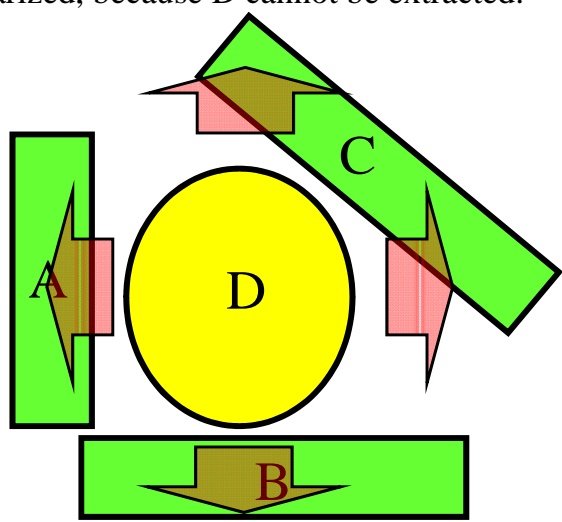

Fig. 4: Inclusion of a component

\section{Implementation}

We developed a module design support system based on the methodology described above. 5):

This system modularizes a product as follows (see Fig.

1) The system imports a three-dimensional geometric model and component connectivity graph of a product (see Fig. 6). The node of the connectivity graph indicates each component and contains the values of its attributes as shown in Table 1.

2) Components are classified into groups by using SOM as described in Section 3.1.

3) The system searches for inclusion relationship as described in Section 3.4.

4) The module structure is constructed so as to maximize the value Modularity. The constructing algorism is in two steps. Initially, each component is considered as an individual module and, therefore, the number of modules equals to the number of components.

I. Calculate Combination $\left(x_{i}, x_{j}\right)$ for all pairs of modules (initially, they are equal to components) in a group.

II. Connect the pair of modules that has highest Combination $\left(x_{i}, x_{j}\right)$ value in to a new module. At the same time, the approximate module structure, represented as the simply connected space of convex hulls, is extended by including the convex hull of the pair. This operation decreases the number of modules by one.

The steps I and II are repeated until Modularity or the number of modules reaches the value that user determined.

Since the computational complex of this algorithm is $\mathrm{O}\left(\mathrm{N}^{2}\right)$, where $\mathrm{N}$ is the number of components, this algorithm is feasible enough.

5) If necessary, the user modifies the design of the product so as to increase the value Modularity. Here, we prepare design modification operations: change of attributes of a component for changing its group to another, change of connectivity of components, and change of position of a component.

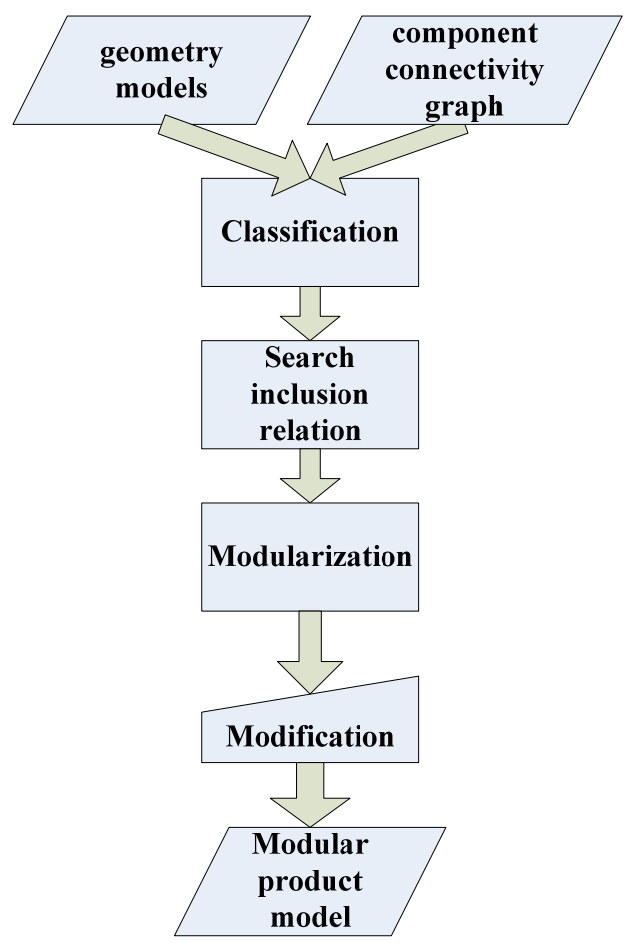

Fig. 5: Flow of modularization

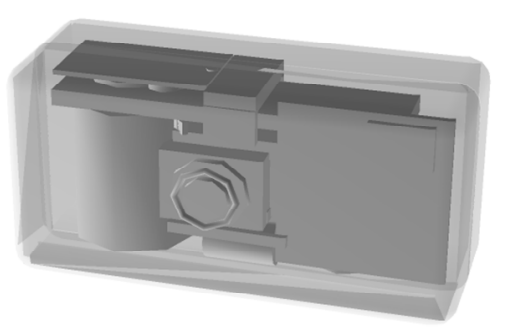

Fig. 6: Geometry model

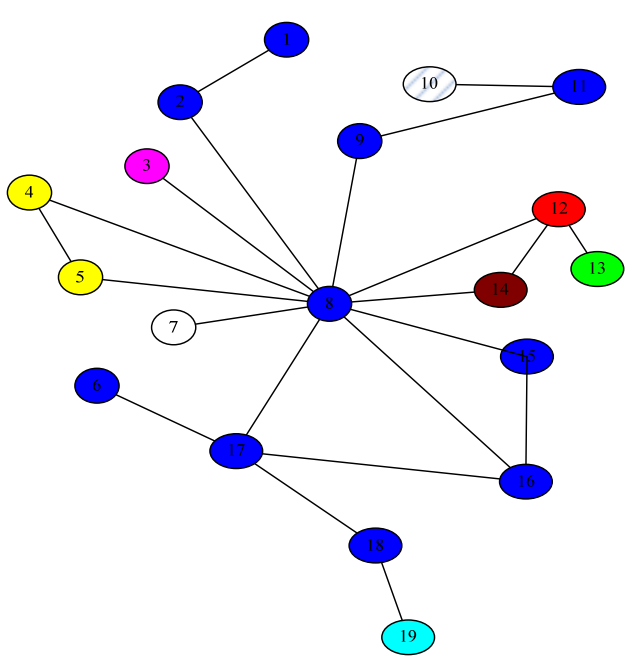

Fig. 7: component connectivity graph 
Table 1: Attributes of components

\begin{tabular}{|c|c|c|c|c|}
\hline & \multicolumn{2}{|c|}{ Life time } & \multirow{2}{*}{ Material } & \multirow{2}{*}{ Life Cycle Option } \\
\hline Name & Phisical & Value & & \\
\hline Counter & 5 & 5 & $\mathrm{PC}, \mathrm{Fe}$ & recycling \\
\hline Spring & 5 & 5 & $\mathrm{Fe}$ & reuse, maintenance \\
\hline Lenz & 7 & 6 & $\mathrm{PC}, \mathrm{Fe}, \mathrm{Cu}$ & reuse \\
\hline Shutter & 5 & 5 & $\mathrm{PC}$ & recycling \\
\hline Roller & 7 & 5 & $\mathrm{Fe}$ & recycling, reuse \\
\hline Strobo Unit & 5 & 5 & $\mathrm{Fe}, \mathrm{Cu}$ & recycling, reuse, upgrade \\
\hline Body & 7 & 5 & $\mathrm{PC}, \mathrm{Fe}$ & recycling \\
\hline Gear & 6 & 5 & $\mathrm{PC}$ & recycling \\
\hline Flont Cover & 5 & 5 & $\mathrm{PC}, \mathrm{Fe}$ & recycling \\
\hline Slide & 6 & 5 & PC & recycling \\
\hline
\end{tabular}

Note that Life time values are relative values, the component that has several materials means that the component consists of all of them, and the component that has several life cycle options means that the one can adopt any of them.

\section{Case study - inkjet printer}

In order to verify the advantages of the methodology, we executed a case study by taking an inkjet printer (see Fig. 8) as an example. The number of components of this printer is 166. Fig. 8 denotes the component connectivity graph; the color of nodes shows the result of classification with SOM and each link shows the physical connections of the components. In the case study, we compared three kind of module structure derived from the same component groups obtained from SOM; (1) the structure derived only with the connectivity of components, (2) the one only with the geometric information, and (3) the one with both of the connectivity and the geometric information.

Fig. 9 shows the number of modules in each case. In this figure, "class change" denotes the module structure modified from the structure (3) by applying the design operation for change of components' groups. Fig. 10 shows the geometry model of the resulting module structure in each case. The number of modules in the structure (1) is 101 . On the other hand, the structure (2) and (3) are 41 and 40, respectively. Therefore, we can see that the number of modules is much reduced by using "module density."

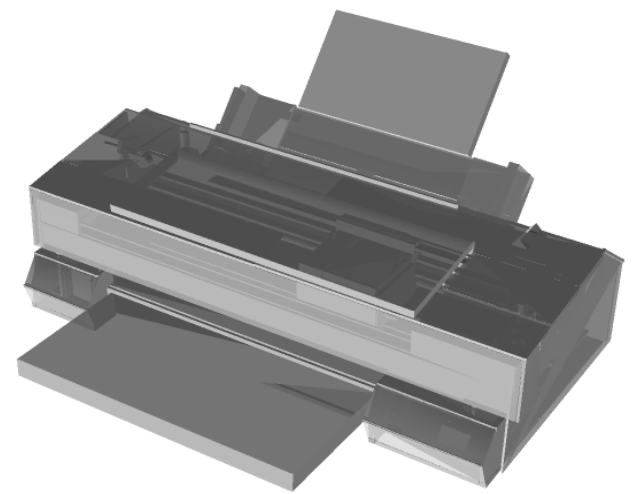

Fig. 7: A geometry model of an inkjet printer(a housing with semitransparency)

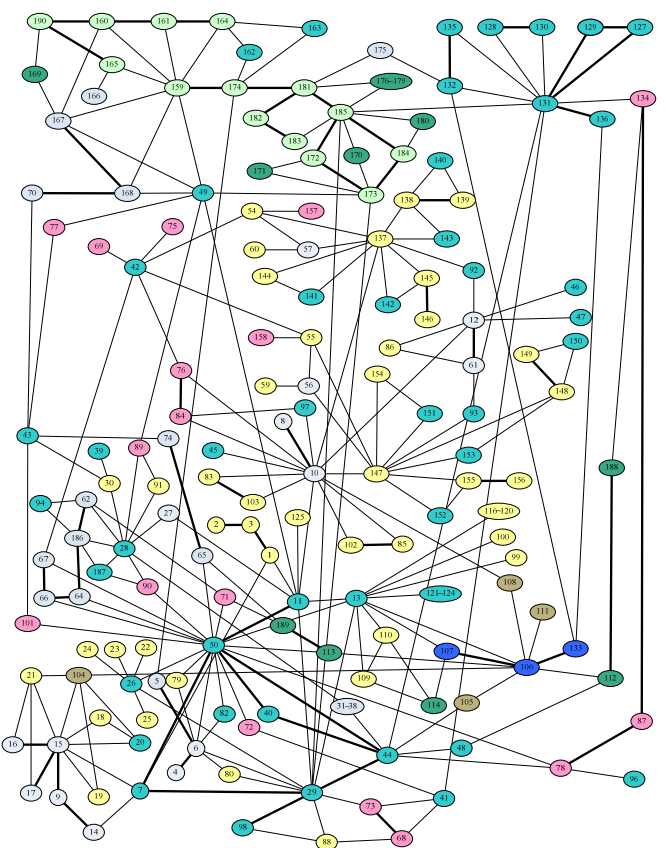

Fig. 8: Component connectivity graph

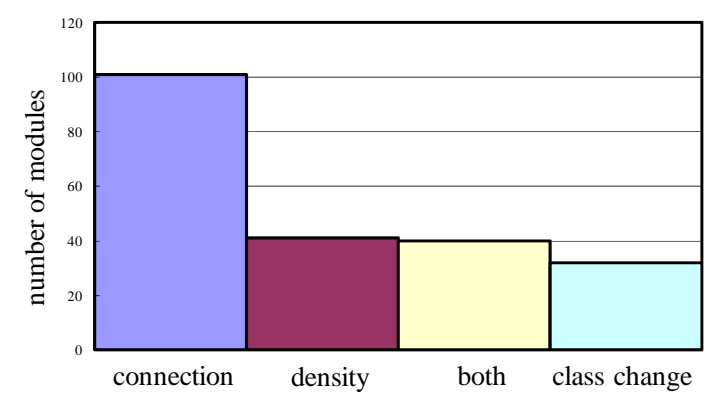

Fig. 9: Number of modules

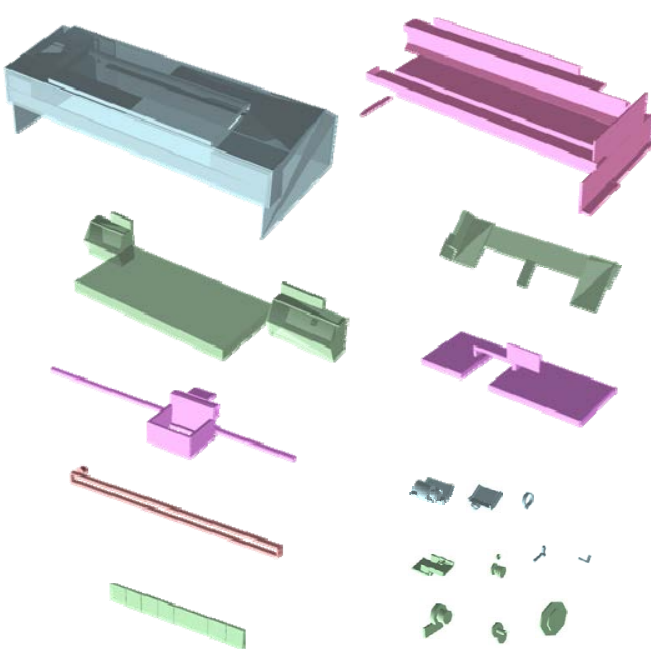

(a) module structure by using only connection relationship 


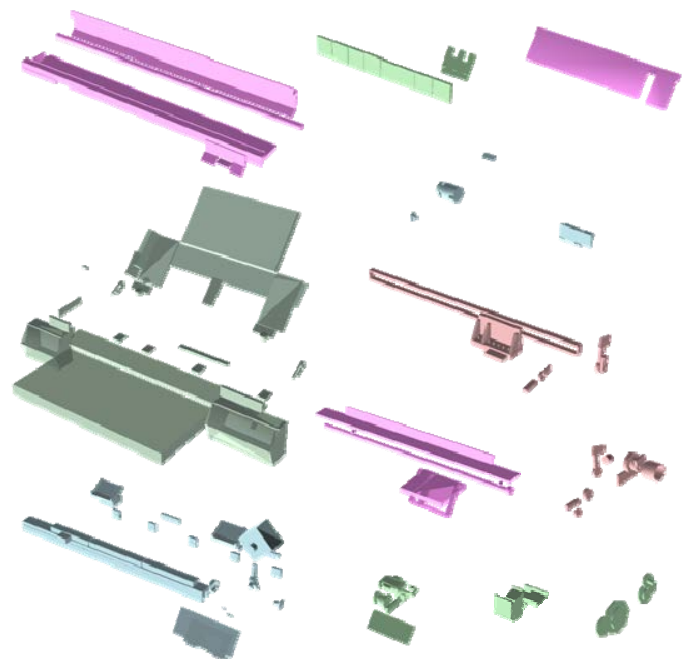

(b) module structure by using only module density

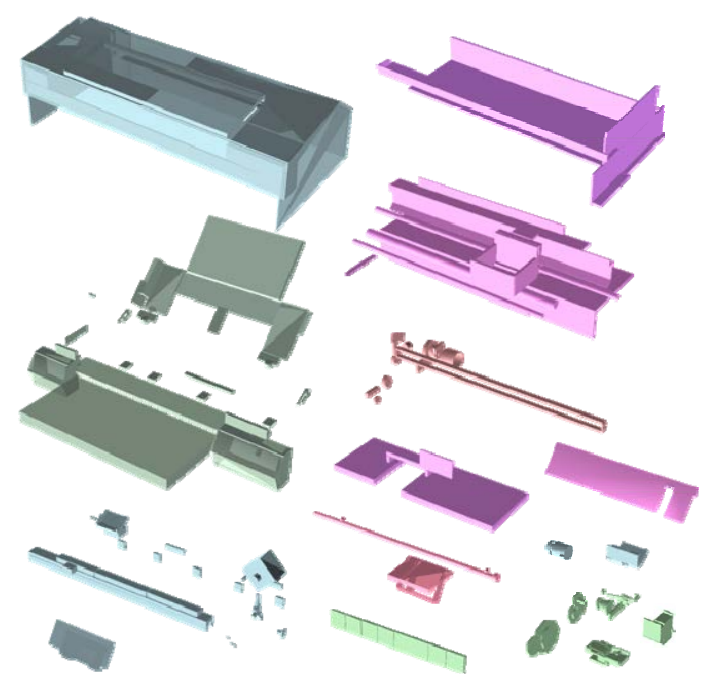

(c) module structure by using both of connection relationship and module density

Fig. 10: Module structure in each case

\section{Discussions}

The result of case study clarified that the structure (1) and (2) are quite different with each other. Since the structure (1) is derived only with the connectivity of components, components in all modules are connected with each other. Therefore, we can realize this structure easily. However, they are restricted by connection relationship; the structure (1) contains only small modules that have few components. On the other hand, the structure (2), which is derived only with the geometric information, contains modules that have components unconnected with each other. By modifying position and shapes of components, we can connect those components. While ignoring existing connection of components means that we can extend modules arbitrarily, the proposed method modularizes the components under the constraint that requires the module density to be high enough. The resulting structure (2) indicates that some of modules are hardly to realize. This means that the constraint of the module density is too weak to derive feasible module structure.
The structure (3) is in between the structure (1) and (2). A designer can adjust the weight of them. In other words, the designer can expand the module structure with keeping the geometric consistency. Therefore, the structure (3) is the most practical (Fig. 11). As a result, we can conclude that the proposed methodology is effective for supporting the module design.

The features of the proposed method are summarized as follows:

1) The method can derive modules with components that do not have a direct connection with each other (see Fig. 12 (c)). There are no existing methods deriving such module structure. A designer can realize such module structure by modifying the design. In this sense, this method gives a hint for module design to a designer.

2) In this method, a designer can control granularity of modules by changing the weight of Modularity parameter and number of groups in the classification.

3) Because the method uses both of geometric information and classification based on components' attributes, the derived module structure is geometrically feasible as well as environmentally conscious from the life cycle viewpoint.

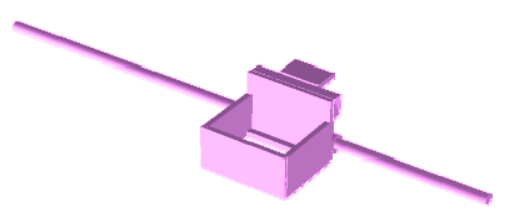

(a) connection relationship

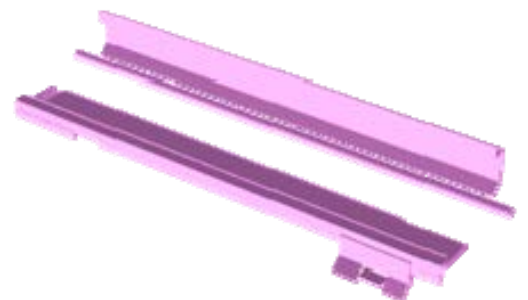

(b) module density

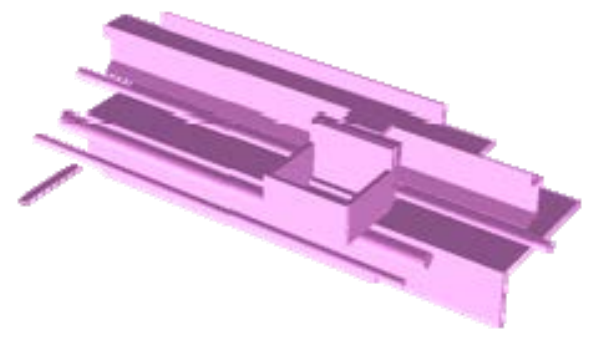

(c) both

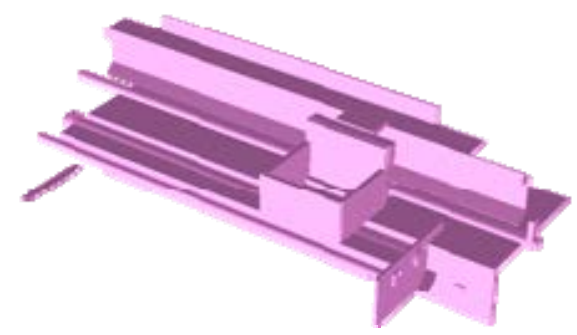

(d) class change

Fig. 11: An example of a module in each case 


\section{Conclusion}

In this paper, we proposed a modular design methodology by which we can derive module structure by using both of environmental aspects and geometric information. This methodology derives the modular structure that is geometrically feasible as well as satisfies the various demands from product life cycle.

The results of this research include:

1) By using module density represented by the convex hull, we derive appropriate module structure from the geometric viewpoint.

2) The case study of an inkjet printer verified feasibility and advantages of the proposed methodology.

We deals mainly with the geometric aspects and the connectivity of the product structures, but the mechanical structures have to be verified from various viewpoints, such as rigidity, deformation and manufacturability. In the future, we plan to evaluate the derived module structure from these viewpoints as well as the environmental aspect of the entire product life cycle, disassemblability, recyclability etc. And support of structural change of products such as shape and position of components is a challenging problem.

\section{Reference}

[1] Seliger, G., Consiglio, S., Odry, D., Zettl, M., 2005, Development of Intelligent Modular Tools for Disassembly, Proceedings of $15^{\text {th }}$ International CIRP Design Seminar 2005, pp. 182-187, (CD-ROM).
[2] Seliger, G., Skerlos, S.J., Basdere, B., Zettl, M., 2003, Design of a Modular Housing Platform for Remanufacturing of Multiple Cellular Phone Models, Proceedings of EcoDesign 2003, pp. 243-250.

[3] Martin, M. V. and Ishii, K, 2002, Design for variety: developing standardized and modularized product platform architectures, Research in Engineering Design, Vol. 13, No. 4, pp. 213-235.

[4] Stone, R., Wood, K., and Crawford, R., 1998, A Heuristic Method to Identify Modules from a Functional Description of a Product, Proceedings of DETC98, DETC98/DTM-5642, (CD-ROM).

[5] Pimmler, T. and Eppinger, S., 1994, Integration Analysis of Product Decompositions, Proceedings of the ASME Design Theory and Methodology Conference, DE-Vol. 68, pp. 343-351.

[6] Kusiak, A., Huang, C.-C., 1997, Design of Modular Digital Circuits for Testability, IEEE Transactions on Components on Components, Packaging and Manufacturing - Part C 20 (1), pp. 48-57.

[7] Shimabukuro, A. Umeda, Y, Kondoh, K, 2004, Development of Modular Design Method for Inverse Manufacturing, Proceedings of the JSPE 2004 Fall Symposium, B73, pp. 157-158. (in Japanese) 